\title{
Semántica bidimensional y teoría de la identidad en Kripke
}

\author{
Two-Dimensional Semantics and the Theory of Identity \\ According to a Kripkeanian Perspective
}

RODRIGO NEIRA CASTAÑN*

\begin{abstract}
Resumen: El presente trabajo consistirá en aplicar el esquema de la semántica bidimensional - la cual se define porque pone en relación convenciones semánticas con mundos posibles y permite dar una respuesta a las ilusiones modales que se originan al asimilar lo contingente a lo $a$ posteriori y lo necesario a lo a priori- a la comprensión de la crítica de Kripke a la teoría de la identidad entre los estados mentales y los estados cerebrales; su conclusión, tomando como ejemplo la identificación del dolor con la estimulación de unas fibras particulares, será que un enunciado que afirme la identidad mente-cerebro no podrá ser necesario, de tal manera que la teoría de la identidad quede en entredicho.

Palabras clave: designador rígido, designador accidental, referencia, mundo posible, contexto de uso.
\end{abstract}

\begin{abstract}
The aim of the current paper consists of applying the conceptual background of the Two-Dimensional Semantics - which allows to reject some modal delusions which have their origins in the assimilation between the contingent and the a posteriori and the necessary and the $a$ priori by connecting semantic conventions with possible worlds - to the understanding of the criticism against the Theory of Identity between mental and physical states put forward by Kripke; its conclusion, which will be arrived at after considering the identification of pain with the stimulation of some particular fibers, will be that the correspondent statement cannot be necessary, so that the Theory of Identity is put into question. Keywords: rigid designator, accidental designator, reference, possible world, context of use.
\end{abstract}

Para Kripke (1985:154-161) todo aquél que defienda la teoría de la identidad entre tipos de estados está comprometido con ciertos supuestos: si el dolor ha de ser la estimulación de las fibras $\mathrm{C}$, tesis que bien podría sostener un teórico de la identidad, entonces esa proposición ha de ser verdadera en todos los mundos posibles y estar constituida por designadores rígidos, expresiones cuyo referente es el mismo en relación a cualesquiera situaciones contrafácticas de las que se hable. Si A es el nombre de una sensación y B el nombre del estado cerebral con el que -según asegura la teoría de la identidad- esa

Fecha de recepción: 11/05/2016. Fecha de aceptación: 20/07/2016.

* Estudiante de doctorado en la Universidad de Oviedo. Correo electrónico: UO228519@uniovi.es . Líneas de investigación: transparencia, externismo de los contenidos mentales, autoridad de primera persona, ficcionalismo. Publicaciones: "La versión modal del argumento ontológico". Textos y estudios de filosofía medieval: De Natura: la naturaleza en la Edad Media. Vol. II., 2015, pp. 653-659; "Recensión de Possibility de M. Jubien". Teorema. Vol. XXXII/3, 2013, pp. 179-183. 
sensación se identifica, entonces A y B deberían ser designadores rígidos y el enunciado A =B debería ser necesariamente verdadero: el estado mental y el estado cerebral serían una y la misma cosa y las propiedades del uno serían las del otro. Ahora bien, este compromiso que debe asumir la teoría de la identidad no parece avenirse con que podamos concebir que B, el estado cerebral, podría haber existido sin que se presentara la sensación A -imaginémonos un caso de anestesia o los efectos derivados de la ingestión de una droga. ¿Cómo puede responder a estos el teórico de la identidad? Desde luego no puede darlo por bueno y seguir argumentando a partir de ahí: los compromisos que ha asumido al adoptar la tesis de la identidad -el carácter necesario de los enunciados de identidad con designadores rígidos- imponen su legalidad teórica y le impiden aceptar sin más la premisa cartesiana a la vez que niega la conclusión cartesiana. Tampoco caben salidas de emergencia como la de argüir que ser tal sensación es una propiedad contingente de A. Pero eso resulta de todo punto inadmisible: ¿acaso ser una determinada sensación es una propiedad contingente de esa sensación?, ¿acaso presentarse como dolor es una propiedad contingente del dolor? Un teórico de la identidad que defendiera la postura de que $\mathrm{B}$ puede darse sin que se dé A debería incluso decir que A es contingentemente una sensación, dado que en principio $B$ puede darse sin que se dé ninguna sensación en absoluto. Recordemos que la teoría de la identidad no afirma que B produzca A sino que A y B son una y la misma cosa. Por eso A y B son designadores rígidos y el enunciado que componen es necesariamente verdadero.

En estas líneas se resume la primera parte de la argumentación de Kripke. La crítica de Kripke ha hecho presa hasta ahora en aquellos teóricos de la identidad que, aun aceptando la premisa cartesiana de que podemos concebir la mente como existente al margen del cuerpo, rechazan la conclusión cartesiana de que la mente es distinta del cuerpo. No hemos hecho de momento ninguna distinción en cuanto a las diversas versiones de las teorías de la identidad. Lo que nos interesa destacar por ahora es lo que a nuestro juicio constituye el núcleo de la crítica de Kripke a estos teóricos de la identidad: Kripke denuncia aquí la primera de las confusiones subyacentes a la tesis de la identidad. Según Kripke, los teóricos de la identidad confunden los enunciados de identidad necesariamente verdaderos del modelo $\mathrm{A}=\mathrm{B}$, en los que ambos términos son designadores rígidos, con los enunciados de identidad contingentemente verdaderos en los que uno de los términos es un designador accidental. Son ejemplos de enunciados del primer tipo "El dolor es la estimulación y las fibras C" y "Descartes es B" -entendiendo B como un designador rígido del cuerpo de Descartes-. Del segundo tipo Kripke cita como ejemplo "Franklin es el inventor de las lentes bifocales". Nótese que en este segundo caso "el inventor de las lentes bifocales" podría haberse referido a otro hombre: el hecho de que en el mundo actual Franklin pueda ser honrado como el inventor de las lentes bifocales no quiere decir que no haya otros mundos posibles en los que fue otro hombre, por ejemplo Descartes, quien inventó las lentes bifocales, o en los que las lentes bifocales no fueron inventadas jamás, sin que por ello Franklin no haya existido en esos otros mundos. En el caso del enunciado "Descartes es B" ninguno de los dos términos de la identidad es como "el inventor de las lentes bifocales": Franklin pudo haber existido aun en los mundos posibles que hemos descrito pero, si Descartes es B, entonces Descartes no puede ser concebido sin B y en ningún mundo posible en el que no exista B podrá existir Descartes. 
La semántica bidimensional puede caracterizarse porque tiene en cuenta que el valor de verdad de las oraciones es uno u otro dependiendo de lo que es el caso en el mundo y también de los valores semánticos que puedan tener. Con respecto a la primera parte de la argumentación de Kripke, el análisis bidimensional puede aplicarse de la siguiente manera: dando por buena la tesis de la identidad resulta que "Descartes es B" es un enunciado verdadero en todos los mundos posibles. Al representarlo en una tabla unidimensional la proposición horizontal debe ser siempre verdadera:

\begin{tabular}{|c|c|c|}
\hline $\mathrm{I}$ & $\mathrm{M} 1$ & $\mathrm{M} 2$ \\
\hline $\mathrm{c} 1$ & $\mathrm{~V}$ & $\mathrm{v}$ \\
\hline
\end{tabular}

Supongamos ahora que, mientras que en M1 están vigentes las convenciones semánticas actuales, en M2 el designador rígido B se usa para referirse al cuerpo de Spinoza en vez de al de Descartes. Tendríamos entonces la tabla bidimensional completa:

\begin{tabular}{|c|c|c|}
\hline II & M1 & M2 \\
\hline $\mathrm{c} 1$ & V & v \\
\hline $\mathrm{c} 2$ & F & f \\
\hline
\end{tabular}

¿Cuál es la diferencia con respecto a 'Franklin es el inventor de las lentes bifocales”? En este caso tenemos un enunciado contingentemente verdadero a priori. A diferencia de lo que ocurre respecto de "Descartes es B", este enunciado no es verdadero en todos los mundos posibles, de ahí que en una celda de la línea horizontal aparezca como verdadero y en la otra aparezca como falso. En cambio, conforme a las convenciones semánticas que rigen en cada mundo posible, el enunciado es siempre verdadero en la diagonal. Supongamos que en M2 el inventor de las lentes bifocales no es Franklin sino Descartes mientras que en c2 utilizamos 'Franklin' para referirnos a Descartes. La tabla bidimensional completa es la siguiente:

\begin{tabular}{|c|c|c|}
\hline III & M1 & M2 \\
\hline $\mathrm{c} 1$ & V & $\mathrm{f}$ \\
\hline $\mathrm{c} 2$ & $\mathrm{~F}$ & $\mathrm{v}$ \\
\hline
\end{tabular}

La segunda de las partes en las que hemos dividido la crítica de Kripke a la tesis de la identidad tiene como objeto una versión concreta de dicha tesis. Se trata de la versión de la teoría que identifica tipos de estados mentales y tipos de estados físicos. Un teórico de la identidad que defendiera esta versión de la teoría podría decir que el dolor no es otra cosa que la estimulación de un cierto tipo de fibras que Kripke llama fibras C. Así, este teórico asentiría al enunciado "'El dolor es la estimulación de las fibras C". Kripke denuncia una confusión semejante a la anterior en este planteamiento. El filósofo que defienda que el dolor es la estimulación de las fibras $\mathrm{C}$ queda inmediatamente comprometido con determinadas tesis que debemos hacer explícitas: en particular el teórico se compromete con el carácter necesario del enunciado 'El dolor es la estimulación de las fibras C'. Así pues, el dolor no puede no ser la estimulación de las fibras $\mathrm{C}$ en ningún mundo posible, cosa que al menos 
a primera vista resulta francamente difícil de admitir. ¿Acaso no podemos imaginarnos con suma facilidad la existencia de seres a quienes la estimulación de las fibras $\mathrm{C}$ no les produzca la misma sensación que a nosotros sino otra muy distinta?

Pero al margen de esta intuición la confusión de la que es víctima el teórico de la identidad como identidad de tipos consiste en suponer que el enunciado "'El dolor es la estimulación de las fibras C" es análogo respecto de enunciados científicos como "El calor es el movimiento de las moléculas". En la teoría de los designadores rígidos estos enunciados son necesariamente verdaderos: los términos de género natural tales como 'calor' son designadores rígidos que se refieren en todo caso a los mismos procesos físicos. Es difícil no ver al mismo tiempo que "el movimiento de las moléculas" es un designador rígido. La tesis usual cae presa de la ilusión de contingencia que producen estos enunciados científicos: confundimos el calor con la sensación de calor. Ahora bien, el hecho de que nosotros sintamos un proceso físico de tal o cual manera depende de nuestra constitución fisiológica y bien podría haber sido el caso de que el universo hubiera estado habitado por seres con otra constitución fisiológica para los que el movimiento de las moléculas se presentara bajo otra apariencia. ¿Diremos entonces que el calor es para esos seres una cosa distinta que para nosotros? La respuesta de Kripke es que el calor es en todo caso el movimiento de las moléculas; lo que es contingente es la manera que tenemos de sentir el calor en tanto que está mediada por nuestro aparato senso-perceptual. Identificamos el calor de manera no rígida, y eso es lo que produce en nosotros una ilusión de contingencia.

¿Podemos explicar de la misma manera la aparente contingencia de la identificación del dolor con la estimulación de las fibras C? Los teóricos de la identidad como identidad de tipos suponen que "El dolor es la estimulación de las fibras C" es análogo a "El calor es el movimiento de las moléculas". Si esto fuera así es de suponer que caeremos a propósito del dolor en la misma ilusión de contingencia que a propósito del calor: así como parece que podría darse el movimiento de las moléculas sin que nadie lo sintiera como calor, parece que podría darse la estimulación de las fibras $\mathrm{C}$ sin que nadie la sintiera como dolor. Recordemos aquí que el teórico de la identidad no afirma que el estado mental sea un efecto producido por el estado físico o un epifenómeno del estado físico, sino que niega toda distancia entre ambos: son una y la misma cosa. Si bien puede haber seres que tengan la sensación de calor aunque no estén en presencia del movimiento de las moléculas, es decir, seres que se encuentren en la misma situación epistémica que nosotros cuando estamos en contacto con el calor objetivo, no parece que el mismo esquema se pueda aplicar al caso del dolor: tener la sensación de dolor es estar en contacto con el dolor objetivo. En el caso del dolor determinamos la referencia de acuerdo con una propiedad esencial de la cosa y no de acuerdo con la propiedad accidental de que produzca en nosotros, dada la mediación de nuestra fisiología, tal o cual sensación. El resultado de estos razonamientos es la conclusión de que la aparente contingencia de la identificación del dolor con un proceso físico no es ilusoria, como en el caso del dolor y su identificación con el movimiento de las moléculas. Por tanto, el teórico de la identidad no puede explicarla de esa manera.

La aspiración de la teoría de la identidad como identidad de tipos es que la tabla resultante del análisis bidimensional de "El dolor es la estimulación de las fibras C" sea como en II, es decir, que arroje como resultado un enunciado verdadero en todo mundo posible. Ese mismo sería el análisis resultante de "El calor es el movimiento de las moléculas". 
En cambio, la tabla resultante si damos por buena la contingencia de la identidad del dolor con la estimulación de las fibras C sería como en III. Supongamos que M1 es el mundo actual mientras que en M2 los únicos seres con conciencia sensible que pueblan el universo experimentan la sensación de dolor -es decir, el dolor mismo- con la estimulación de las fibras $\mathrm{H}$ en vez de C. En c2 la convención semántica que rige es la de referirse con 'dolor' a la estimulación de las fibras $\mathrm{H}$ :

\begin{tabular}{|c|c|c|}
\hline IV & M1 & M2 \\
\hline $\mathrm{c} 1$ & V & f \\
\hline $\mathrm{c} 2$ & F & v \\
\hline
\end{tabular}

Se trata pues de un enunciado contingentemente verdadero a priori. Esto implica que es posible un estado físico sin el estado mental correspondiente en el mundo actual. Si esa correspondencia es contingente, la tesis de la identidad queda en entredicho.

\section{Referencia bibliográfica}

Kripke, Saul (1985): El nombrar y la necesidad, Universidad Nacional Autónoma de México, México D.F. 
